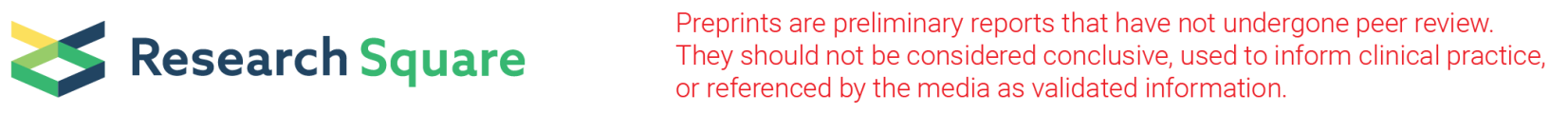

\title{
Effectiveness of Patient-centered Care for Diabetes Self-management to Improve Glycemic Control and Self-care Behaviors in Adults With Type 2 Diabetes: a Systematic Review
}

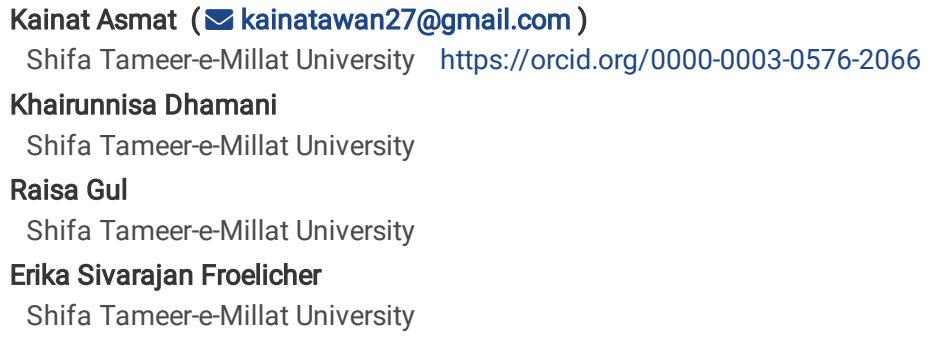

Research

Keywords: Education and counselling, HbA1c, meta-analysis, patient-centred care, type 2 diabetes, self-management

Posted Date: July 8th, 2021

DOI: https://doi.org/10.21203/rs.3.rs-674310/v1

License: (a) (1) This work is licensed under a Creative Commons Attribution 4.0 International License. Read Full License 


\section{Abstract}

Background: Patient-centered care in diabetes self-management might be a significant factor in improving self-care outcomes yet the supporting evidence is inadequate. This review is aimed to assess the effectiveness of patient-centered self-management care interventions on self-care outcomes such as glycemic control $(\mathrm{HbA} 1 \mathrm{c})$ and self-care behaviors in adults with type-2 diabetes compared with usual care.

Methods: CINAHL, PubMed, Cochrane Library, Google Scholar and the HEC Pakistan digital library were searched for English language studies that assessed patient-centered self-management educational and/or behavioral interventions in adults aged 18 years or above with type 2 diabetes from 1991 to 2020. Interventional studies comprising randomized controlled trials (RCT) and quasi experimental studies (QES) with at least three months follow up and reporting on self-care outcomes with glycemic control ( $\mathrm{HbA} 1 \mathrm{c})$ as primary outcome and self-care behaviors including diet control, physical activity, medication adherence and foot care as secondary outcomes were included.

Results: Of the 168 identified records, 25 were found eligible comprising 21 RCTs and 4 QESs with total 4,443 participants. The meta-analysis involved 23 studies that provided enough information for a pooled estimate of HbA1c. Compared with the control group, patient-centered self-management interventions significantly lowered $\mathrm{HbA} 1 \mathrm{c}-0.53(95 \% \mathrm{Cl}-0.73,-0.32)$. Stratified analysis for $\mathrm{HbA} 1 \mathrm{c}$ with respect to various aspects of intervention showed larger effects in interventions employing both educational and behavioral components $-0.59(95 \% \mathrm{Cl}-0.86,-0.32)$, spanned over shorter (<03 months) duration $-0.56(95 \% \mathrm{Cl}$ $-0.86,-0.27)$, administered by nurses $-0.80(95 \% \mathrm{Cl}-1.44,-0.16)$ and delivered in community setting $-0.65(95 \% \mathrm{Cl}-1.00,-0.29)$. Moreover, patient-centered self-management interventions were found effective in improving diet control, physical activity and foot care.

Conclusion: This systematic review provided the evidence supporting the effectiveness of patient-centered self-management care interventions in improving glycemic control and self-care behaviors in adults with type 2 diabetes and identified key features of intervention contributing towards success.

\section{Background}

Diabetes mellitus (DM) refers to a group of metabolic conditions characterized by unusual high blood glucose levels with impairment in carbohydrates, protein and fat metabolism due to abnormalities in insulin secretion, insulin response or both(1). World Health Organization (WHO) devides DM into two types: type 1 and type 2(1). Type 1 DM is characterised by immune mediated beta cell destruction that leads to absolute insulin deficiency(2). Type 2 DM implies gradual loss of beta cell activity resulting in relative insulin deficieny(2). Hyperglycaemia is the classical clinical manifestation in both types that is mainly linked with an increased risk of microvascular- and macrovascular complications(3). Microvascular complications such as neuropathy, retinopathy and nephropathy as well as macrovascular complications like stroke, ischaemic heart disease and peripheral vascular disease; are chief contributors of increased morbidity and mortality in this population(4).

According to Interntional Diabetes Federation (IDF) report 2019, "463 million people (aged 20-79 years) worldwide have DM" where type 2 DM accounts for $90 \%$. Furthermore, it is projected that by the year 2045, 700 million people will have DM (5). Based on IDF, the Middle East and North Africa region has a high prevalence of DM, with 55 million people affected(5). Furthermore, it has been reported that the incidence is highest among adults living in low- and middleincome countries, accounting for $79 \%$ of all cases (6).

Pakistan is a low middle income country with an estimated 221.7 million population making it the world's sixth most populous country(7). Pakistan ranks fourth among top ten countries with the highest number of DM population aged 20-79 years (5). As per IDF report, Pakistan has 19.4 million people (aged 20-79 years) with DM and it is estimated that by $2045,37.1$ million will have DM moving Pakistan to third place (5). Another study estimates that the current DM prevalence in Pakistan is around $26.9 \%$ with type 2 DM accounting for $90 \%$ of all cases(8).

The rise in type 2 diabetes in Pakistan is driven by socioeconomic, demographic, environmental and genetic factors with key contributors including growing urbanisation, sedentary lifestyle, unhealthy eating habits and obesity (3). Besides, a poor health care system including inaccessibility to health care centers, inequality in health care services provision, gender disparity and poor socioeconomic conditions with low level education as well as unemployment are aggravating the problem (9).

Type 2 DM due to its growing prevalence has turned into a serious health problem for Pakistan and around the world. If not properly managed, it is posing a substantial risk to morbidity and mortality as a result of devastating health complications(10). On the other hand, it is increasing the financial burden of individuals and their families with significant impact on the health systems and national economies (11). Currently, Pakistan is spending $12 \%$ of its total health budget (less than $1 \%$ of gross domestic product) on DM care with nearly $70 \%$ devoted to the management of DM associated complications (9). Therefore, delaying the progression of DM and avoiding its associated complications is the cornerstone that can lead to improved health and economic outcomes of individuals, families, society and the health care system (12).

According to established guidelines, a healthy lifestyle with improvement in self-care behaviours and medication as needed; can delay type 2 DM progression and thus, avoivd serious complications(13). As a result, type $2 \mathrm{DM}$, is also known as a self-managed condition because patient themselves perform majority of their care(14). Effective self management requires patient's full commitment and capability to perform self-care activities such as healthy dietary habits, daily physical activity, smoking cessation, regular blood glucose monitoring and regular intake of medicines (12). Patient needs to make a concerted and self motivated effort towards adoption of a healthy life style as pharmacotherapy alone cannot achieve these goals(15). Therefore, for effective self management of type 2 DM; a patient-centered approach is certainly needed.

Patient-centered care has been acknowledged as a desirable attribute of health care since late 1980s when the concept 'patient centeredness' was first introduced(16). Patient centeredness refers to the use of a bio-psycho-social perspective which means focusing on the patient and honoring his/her preferences, needs and values as a holistic being rather than a biomedical perspective which focuses on disease(17). Patient-centered care in type 2 DM self- 
management is a purposefully designed holistic care intervention that provides individuals with information and skills they need to effectively self-manage their condition and achieve optimum glycemic control in addition to medication (18). Self-management education is the major component of patient-centered care which according to WHO, provides basis for management of the disease(19). The literature supports that up to $8 \%$ of diabetes-assoicated complications can be reduced through proper self-management education(20). Counselling as behavioral intervention is another major component of patient-centred care. The American Association of Diabetes Educators (AADE) suggests to prepare patients for behaviour change by epuipping them with the necessary skills to improve their self-care behaviors. According to $A A D E$, seven parameters of self care behaviors are "healthy diet, regular blood-sugar monitoring, regular physical activity, medication adherence, resilient coping skills, effective problem-solving and risk-reduction behaviors (21). Literature supports that behavioral interventions focusing these self care activities resulted in improved health outcomes in this population(22, 23). A meta analysis published in 2003 has demonstrated the effectiveness of educational and behavioral interventions in improving glycemic control in patients with type 2 DM (24).

Given the increasing prevalence of type 2 DM in Pakistan and the risk of increased morbidity and mortality, a patient-centred care can play a crucial role in effective self management. Therefore, an updated systematic review of patient-centered care employing educational and behavioral interventions, would give a better understanding of whether this care approach is associated with improved self-care outcomes. This review aimed to assess the effectiveness of patient-centered care for diabetes self-management to improve glycemic control and self-care behaviors in adults with type 2 DM compared with usual care.

\section{Methods}

\subsection{Search Strategy:}

The literature search was performed in CINAHL, PubMed, Cochrane Library, Google Scholar and the HEC Pakistan digital library for studies in English language published between January 1, 1990 and September 30, 2020. January 1, 1990 was selected as the search initiation date because the term patient-centeredcare/ patient centeredness has been introduced in the literature in late 1980s(16). The Medical Subject Headings (MeSH) terms used were: "Type 2 Diabetes" or "Type II Diabetes," and "Patient-Centered-Care" or "Person-Oriented-Care" or "Holistic Care" or "Self-Care," and "Self-Management" and "Glycemic Control" or "HbA1c". The retrieved titles and abstracts were evaluated for relevance. Articles found relavant were reviewed as full text for consideration of inclusion in this review by completing the eligibility form based on inclusion criteria. In addition to systematic database searches, manual search was performed to find studies in reference lists of relevant articles. This review was planned, carried out and reported in compliance with "preferred reporting items for systematic reviews and meta-analysis (PRISMA) guidelines" (25). Figure. 1 shows the PRISMA flow chart for selection of the studies and reasons for exclusion.

\subsection{Inclusion and Exclusion Criteria:}

Studies that satisfied the inclusion criteria listed below, were found eligible: (1) type of studies as interventional studies comprising RCTs and QESs; (2) type of participants as adults aged 18 years or above diagnosed with type $2 \mathrm{DM}$ for at least last six months; (3) type of intervention as patient-centred care intervention for diabetes self-management with educational and/or behavioral component provided in any setting, by any method, by any provider, for any contact time and follow up for at least three months; (4) comparision intervention as usual care or standard care; lastly, (5) type of outcomes involving glycemic control ( $\mathrm{HbA1c}$ ) as primary outcome and self-care behaviors including diet control, physical activity, medication adherence and foot care as secondary outcomes.

The following studies were excluded: (1) review, (2) non-intervention study, (3) qualitative study, (4) protocol, (5) involved patients with type 1 DM only, (6) involved adult patients with type 2 DM and with other chronic conditions, (7) involved patients under 18 years of age, (8) targeted exclusively at prevention of type 2 DM. and (9) primarily reporting development or feasibility of intervention.

\subsection{Data Extraction}

Data from the eligible studies was extracted and entered into Excel sheet. Data entered was double checked for correctness and completeness. Inconsistencies in retrieved data were adressed and disagreements adjudicated by reaching consensus.

\subsection{Quality Assessment of Individual Studies:}

Methodological quality of Individual studies was assessed using 'Cochrane Collaboration risk of bias (Rob)' assessment tools that yield judgment as low, high or unclear risk. Rob 2 (version 2 of the Cochrane risk of bias tool for randomized trials) was used for RCTs(26). Whereas, ROBINS-I (Risk of bias in nonrandomized studies of Interventions) was used for QESs(27). Blinding of the participants and providers was not possible due to the nature of the intervention. Authors of the studies were contacted to request additional information. Out of twenty one RCTs, nine were graded as low risk of bias (28-36); eight as high risk of bias $(23,37-43)$ and, four as unclear risk of bias $(44-47)$. Out of four QESs; two were of unclear risk of bias $(22,48)$ and the other two were of high risk of bias $(49,50)$.

\section{Statistical Analysis:}

A meta-analysis was performed using REVMAN 5.4.1 to calculate the magnitude of pooled effect size for change in HbA1c, the primary outcome (51). Of 25 included studies, 23 that reported on HbA1c were included in meta-analysis comprising 20 RCTs and 3 QESs. Data entered in REVMAN involved final values of mean and standard deviation of HbA1c for experimental group as well as control group and the number of participants in each group. Standardized mean difference of $\mathrm{HbA} 1 \mathrm{c}$ between experimental and control group and $95 \%$ confidence intervals were calculated for estimation of effect size. $I^{2}$ statistics were used to estimate statistical heterogeneity among studies. Random effect model was applied on more than $50 \%$ heterogeneity(52). To further explore sources of heterogeneity, following stratified analyses were performed: (1) stratified analysis based on quality of the studies; (2) stratified analysis based on key aspects of intervention including (a) component of intervention (educational vs. educational and behavioral), (b) duration of intervention (< three month vs. three to six 
months vs. > six months), (c) provider of intervention (nurse vs. other professional vs. $\geq 2$ disciplines) and (d) setting of intervention (hospital vs. community vs. combined hospital and community).

\section{Results}

\subsection{Characteristics of Studies:}

For this review, 25 studies met the eligibility criteria. The included studies were published between 1998 and 2020 where the majority (60\%) of the studies was published between 2015 and 2020. Most of the studies were RCTs accounting for $84 \%(23,29-34,36-39,41-47,53-55)$. Total 4,443 participants were involved with the mean age of 56.1 years (range 18 to 69 years). Study population involved patients with type 2 DM with mean duration of disease $7.5 y e a r s$ (range 06 months (42) to 12.9 years (46)). Single study sample size ranged from $22(56)$ to $886(30)$ where the sample size in each study involved both male and female patients. The characteristics of 25 studies are presented in Table 1. 
Table 1

Characteristics of 25 included studies in the review

\begin{tabular}{|c|c|c|c|c|c|c|c|c|c|c|}
\hline \multirow[t]{2}{*}{ No. } & \multirow{2}{*}{$\begin{array}{l}\text { Author \& } \\
\text { Year }\end{array}$} & \multirow[t]{2}{*}{ Country } & \multirow{2}{*}{$\begin{array}{l}\text { Study } \\
\text { Design }\end{array}$} & \multirow{2}{*}{$\begin{array}{l}\text { No. of } \\
\text { Participants } \\
\text { recruited / } \\
\text { at follow up }\end{array}$} & \multicolumn{6}{|l|}{ Intervention } \\
\hline & & & & & $\begin{array}{l}\text { Theoretical } \\
\text { Basis }\end{array}$ & $\begin{array}{l}\text { Mode of } \\
\text { delivery }\end{array}$ & Provider & Setting & Components & Duri \\
\hline \multirow[t]{2}{*}{1} & \multirow{2}{*}{$\begin{array}{l}\text { Kinmonth } \\
\text { et al. }\end{array}$} & \multirow[t]{2}{*}{ England } & \multirow[t]{2}{*}{$\mathrm{RCT}$} & \multirow[t]{2}{*}{$360 / 250$} & \multirow[t]{2}{*}{ NR } & \multirow{2}{*}{$\begin{array}{l}\text { Face to } \\
\text { Face }\end{array}$} & Nurse & \multirow[t]{2}{*}{ Hospital } & Educational & \multirow[t]{2}{*}{ NR } \\
\hline & & & & & & & Physician & & Behavioral & \\
\hline 2 & $\begin{array}{l}\text { Glasgow et } \\
\text { al. } 20\end{array}$ & $\begin{array}{l}\text { Colorado, } \\
\text { USA }\end{array}$ & $\mathrm{RCT}$ & $886 / 733$ & NR & $\begin{array}{l}\text { Face to } \\
\text { Face }\end{array}$ & Physician & Hospital & $\begin{array}{l}\text { Educational } \\
\text { Behavioral }\end{array}$ & $\begin{array}{l}>06 \\
\text { Mor }\end{array}$ \\
\hline 3 & Tayler et al. & Canada & RCT & $40 / 39$ & $\begin{array}{l}\text { Supportive } \\
\text { Care Model }\end{array}$ & $\begin{array}{l}\text { Face to } \\
\text { Face }\end{array}$ & $\begin{array}{l}\text { Nurses } \\
\text { Physician }\end{array}$ & Community & $\begin{array}{l}\text { Educational } \\
\text { Behavioral }\end{array}$ & $\begin{array}{l}04 \\
\text { Mo }\end{array}$ \\
\hline 4 & Scain et al. & Brazil & $\mathrm{RCT}$ & $104 / 104$ & NR & $\begin{array}{l}\text { Face to } \\
\text { Face }\end{array}$ & Nurse & Hospital & Educational & $\begin{array}{l}04 \\
\text { wee }\end{array}$ \\
\hline 5 & $\begin{array}{l}\text { Sacco et al. } \\
21\end{array}$ & Florida, USA & $\mathrm{RCT}$ & $62 / 48$ & NR & Telephone & Psychologist & Community & Educational & $\begin{array}{l}06 \\
\text { Mor }\end{array}$ \\
\hline 6 & Carter et al. & $\begin{array}{l}\text { Washington, } \\
\text { USA }\end{array}$ & RCT & $74 / 47$ & NR & Online & Nurse & Community & $\begin{array}{l}\text { Educational } \\
\text { Behavioral }\end{array}$ & NR \\
\hline 7 & $\begin{array}{l}\text { Forjuoh et } \\
\text { al. }\end{array}$ & Texas, USA & RCT & $376 / 263$ & NR & $\begin{array}{l}\text { Face to } \\
\text { face }\end{array}$ & Physician & $\begin{array}{l}\text { Hospital } \\
\text { and } \\
\text { Community }\end{array}$ & $\begin{array}{l}\text { Educational } \\
\text { Behavioral }\end{array}$ & $\begin{array}{l}06 \\
\text { Wee }\end{array}$ \\
\hline 8 & Yuan et al. & $\begin{array}{l}\text { Hong Kong, } \\
\text { China }\end{array}$ & $\mathrm{RCT}$ & $88 / 76$ & NR & $\begin{array}{l}\text { Face to } \\
\text { face }\end{array}$ & Nutritionist & Hospital & Educational & $\begin{array}{l}08 \\
\text { wee }\end{array}$ \\
\hline
\end{tabular}




\begin{tabular}{|c|c|c|c|c|c|c|c|c|c|c|}
\hline \multirow[t]{2}{*}{ No. } & \multirow{2}{*}{$\begin{array}{l}\text { Author \& } \\
\text { Year }\end{array}$} & \multirow[t]{2}{*}{ Country } & \multirow{2}{*}{$\begin{array}{l}\text { Study } \\
\text { Design }\end{array}$} & \multirow{2}{*}{$\begin{array}{l}\text { No. of } \\
\text { Participants } \\
\text { recruited / } \\
\text { at follow up }\end{array}$} & \multicolumn{6}{|l|}{ Intervention } \\
\hline & & & & & $\begin{array}{l}\text { Theoretical } \\
\text { Basis }\end{array}$ & $\begin{array}{l}\text { Mode of } \\
\text { delivery }\end{array}$ & Provider & Setting & Components & Duri \\
\hline 9 & $\begin{array}{l}\text { Escamilla } \\
\text { et al. }\end{array}$ & $\begin{array}{l}\text { Connecticut, } \\
\text { USA }\end{array}$ & $\mathrm{RCT}$ & $211 / 148$ & NR & $\begin{array}{l}\text { Face to } \\
\text { face }\end{array}$ & $\begin{array}{l}\text { Community } \\
\text { Health Worker } \\
\text { (CHW) }\end{array}$ & Community & $\begin{array}{l}\text { Educational } \\
\text { Behavioral }\end{array}$ & $\begin{array}{l}12 \\
\text { mor }\end{array}$ \\
\hline \multirow[t]{3}{*}{10} & \multirow{3}{*}{$\begin{array}{l}\text { Ebrahimi et } \\
\text { al. }\end{array}$} & \multirow[t]{3}{*}{ Iran } & \multirow[t]{3}{*}{ RCT } & \multirow[t]{3}{*}{$106 / 103$} & \multirow{3}{*}{$\begin{array}{l}\text { Empowerment } \\
\text { Model }\end{array}$} & \multirow{3}{*}{$\begin{array}{l}\text { Face to } \\
\text { face }\end{array}$} & Nurse & Hospital & \multirow{3}{*}{$\begin{array}{l}\text { Educational } \\
\text { Behavioral }\end{array}$} & \multirow{3}{*}{$\begin{array}{l}08 \\
\text { wee }\end{array}$} \\
\hline & & & & & & & Endocrinologist & & & \\
\hline & & & & & & & Nutritionist & & & \\
\hline 11 & $\begin{array}{l}\text { Jutterström } \\
\text { et al. }\end{array}$ & Sweden & RCT & $195 / 171$ & $\begin{array}{l}\text { Hernandez } \\
\text { theory of } \\
\text { integration }\end{array}$ & $\begin{array}{l}\text { Face to } \\
\text { face }\end{array}$ & Nurse & Hospital & $\begin{array}{l}\text { Educational } \\
\text { Behavioral }\end{array}$ & $\begin{array}{l}06 \\
\text { Mor }\end{array}$ \\
\hline 12 & $\begin{array}{l}\text { Windrum. } \\
\text { et al. }{ }^{19}\end{array}$ & $\begin{array}{l}\text { Hampshire, } \\
\text { UK }\end{array}$ & $\mathrm{RCT}$ & $203 / 203$ & NR & $\begin{array}{l}\text { Face to } \\
\text { face }\end{array}$ & Physician & Hospital & Educational & $\begin{array}{l}03 \\
\text { Wee }\end{array}$ \\
\hline 13 & $\begin{array}{l}\text { Azami et } \\
\text { al. }\end{array}$ & Iran & $\mathrm{RCT}$ & $142 / 142$ & $\begin{array}{l}\text { Social } \\
\text { Cognitive } \\
\text { Theory }\end{array}$ & $\begin{array}{l}\text { Face to } \\
\text { face and } \\
\text { telephone }\end{array}$ & Nurse & Hospital & $\begin{array}{l}\text { Educational } \\
\text { Behavioral }\end{array}$ & $\begin{array}{l}03 \\
\text { Mor }\end{array}$ \\
\hline 14 & $\begin{array}{l}\text { Abraham et } \\
\text { al. }\end{array}$ & India & RCT & $80 / 41$ & NR & $\begin{array}{l}\text { Face to } \\
\text { face and } \\
\text { telephone }\end{array}$ & Physician & Hospital & $\begin{array}{l}\text { Educational } \\
\text { Behavioral }\end{array}$ & $\begin{array}{l}06 \\
\text { wee }\end{array}$ \\
\hline 15 & $\begin{array}{l}\text { Cheng et } \\
\text { al. }\end{array}$ & China & RCT & $242 / 201$ & $\begin{array}{l}\text { Empowerment } \\
\text { Model }\end{array}$ & $\begin{array}{l}\text { Face to } \\
\text { face and } \\
\text { telephone }\end{array}$ & Nurse & Hospital & $\begin{array}{l}\text { Educational } \\
\text { Behavioral }\end{array}$ & $\begin{array}{l}06 \\
\text { wee }\end{array}$ \\
\hline 16 & $\begin{array}{l}\text { Zheng et } \\
\text { al. }\end{array}$ & China & $\mathrm{RCT}$ & $60 / 60$ & NR & $\begin{array}{l}\text { Face to } \\
\text { face }\end{array}$ & Physician & Hospital & $\begin{array}{l}\text { Educational } \\
\text { Behavioral }\end{array}$ & $\begin{array}{l}02 \\
\text { Wee }\end{array}$ \\
\hline
\end{tabular}




\begin{tabular}{|c|c|c|c|c|c|c|c|c|c|c|}
\hline \multirow[t]{2}{*}{ No. } & \multirow{2}{*}{$\begin{array}{l}\text { Author \& } \\
\text { Year }\end{array}$} & \multirow[t]{2}{*}{ Country } & \multirow{2}{*}{$\begin{array}{l}\text { Study } \\
\text { Design }\end{array}$} & \multirow{2}{*}{$\begin{array}{l}\text { No. of } \\
\text { Participants } \\
\text { recruited / } \\
\text { at follow up }\end{array}$} & \multicolumn{6}{|l|}{ Intervention } \\
\hline & & & & & $\begin{array}{l}\text { Theoretical } \\
\text { Basis }\end{array}$ & $\begin{array}{l}\text { Mode of } \\
\text { delivery }\end{array}$ & Provider & Setting & Components & Duri \\
\hline \multirow[t]{3}{*}{17} & Ing et al. & $\begin{array}{l}\text { Honolulu, } \\
\text { USA }\end{array}$ & RCT & $65 / 38$ & NR & $\begin{array}{l}\text { Face to } \\
\text { face }\end{array}$ & $\begin{array}{l}\text { CHW, } \\
\text { Pharmacist, } \\
\text { Nutritionist }\end{array}$ & Community & $\begin{array}{l}\text { Educational } \\
\text { Behavioral }\end{array}$ & $\begin{array}{l}03 \\
\text { Mor }\end{array}$ \\
\hline & & & & & & & Physician & & & \\
\hline & & & & & & & Psychologist & & & \\
\hline 18 & $\begin{array}{l}\text { Varming et } \\
\text { al. }\end{array}$ & Denmark & RCT & $154 / 97$ & NR & $\begin{array}{l}\text { Face to } \\
\text { face and } \\
\text { telephone }\end{array}$ & Nurse & Hospital & $\begin{array}{l}\text { Educational } \\
\text { Behavioral }\end{array}$ & $\begin{array}{l}03 \\
\text { Mor }\end{array}$ \\
\hline 19 & $\begin{array}{l}\text { Spencer et } \\
\text { al. }\end{array}$ & Canada & RCT & $222 / 147$ & NR & $\begin{array}{l}\text { Face to } \\
\text { face }\end{array}$ & $\mathrm{CHW}$ & Community & $\begin{array}{l}\text { Educational } \\
\text { Behavioral }\end{array}$ & $\begin{array}{l}06 \\
\text { Mor }\end{array}$ \\
\hline 20 & Omer et al. & Ajman, UAE & RCT & $218 / 164$ & NR & $\begin{array}{l}\text { Telephone } \\
\text { (WhatsApp }\end{array}$ & Pharmacist & Community & Educational & $\begin{array}{l}06 \\
\text { Mor }\end{array}$ \\
\hline 21 & Utz et. al. & $\begin{array}{l}\text { Virginia, } \\
\text { USA }\end{array}$ & $\begin{array}{l}\text { Quasi } \\
\text { experimental } \\
\text { study }\end{array}$ & $22 / 21$ & $\begin{array}{l}\text { Social } \\
\text { Cognitive } \\
\text { Theory }\end{array}$ & $\begin{array}{l}\text { Face to } \\
\text { Face }\end{array}$ & $\begin{array}{l}\text { Certified } \\
\text { Diabetes } \\
\text { Educator (CDE) }\end{array}$ & Community & $\begin{array}{l}\text { Educational } \\
\text { Behavioral }\end{array}$ & $\begin{array}{l}08 \\
\text { Wee }\end{array}$ \\
\hline 22 & $\begin{array}{l}\text { Gavgani et } \\
\text { al. }\end{array}$ & Iran & $\begin{array}{l}\text { Quasi } \\
\text { experimental } \\
\text { study }\end{array}$ & $32 / 30$ & $\begin{array}{l}\text { Information } \\
\text { Motivation } \\
\text { and } \\
\text { Behavioral } \\
\text { skill Model }\end{array}$ & $\begin{array}{l}\text { Face to } \\
\text { face }\end{array}$ & Physician & Hospital & $\begin{array}{l}\text { Educational } \\
\text { Behavioral }\end{array}$ & $\begin{array}{l}04 \\
\text { Wee }\end{array}$ \\
\hline 23 & $\begin{array}{l}\text { Fardazar et } \\
\text { al. }\end{array}$ & Iran & $\begin{array}{l}\text { Quasi } \\
\text { experimental } \\
\text { study }\end{array}$ & $180 / 180$ & $\begin{array}{l}\text { attribution } \\
\text { theory }\end{array}$ & $\begin{array}{l}\text { Face to } \\
\text { face }\end{array}$ & Physician & Hospital & Educational & $\begin{array}{l}\text { Thre } \\
(60 \\
\text { sess }\end{array}$ \\
\hline 24 & Guner et al. & Turkey & $\begin{array}{l}\text { Quasi } \\
\text { experimental } \\
\text { study }\end{array}$ & $101 / 101$ & NR & $\begin{array}{l}\text { Face to } \\
\text { face and } \\
\text { Telephone }\end{array}$ & $\begin{array}{l}\text { Nurse } \\
\text { Physician }\end{array}$ & Community & Educational & $\begin{array}{l}02 \\
\text { wee } \\
\text { sess } \\
\text { follc } \\
\text { by } S \\
\text { twic } \\
\text { wee } \\
\text { six } \\
\text { mor }\end{array}$ \\
\hline
\end{tabular}




\begin{tabular}{|c|c|c|c|c|c|c|c|c|c|c|}
\hline \multirow[t]{2}{*}{ No. } & \multirow{2}{*}{$\begin{array}{l}\text { Author \& } \\
\text { Year }\end{array}$} & \multirow[t]{2}{*}{ Country } & \multirow{2}{*}{$\begin{array}{l}\text { Study } \\
\text { Design }\end{array}$} & \multirow{2}{*}{$\begin{array}{l}\text { No. of } \\
\text { Participants } \\
\text { recruited / } \\
\text { at follow up }\end{array}$} & \multicolumn{6}{|l|}{ Intervention } \\
\hline & & & & & $\begin{array}{l}\text { Theoretical } \\
\text { Basis }\end{array}$ & $\begin{array}{l}\text { Mode of } \\
\text { delivery }\end{array}$ & Provider & Setting & Components & Duri \\
\hline 25 & Hailu et al. & Ethiopia & RCT & $220 / 142$ & NR & $\begin{array}{l}\text { Face to } \\
\text { face }\end{array}$ & Nurse & Hospital & Educational & $\begin{array}{l}06 \\
\text { Mor }\end{array}$ \\
\hline
\end{tabular}

\subsection{Characteristics of Intervention:}

Interventions of all studies were compared to usual care. Usual care majorly involved consultation with the physician; having blood sugar, blood pressure and weight checked; and getting schedule for the next appointment. $32 \%$ studies ( 8 out of 25 ) reported on theoretical model used for design and implementation of self-management intervention $(22,23,29,33,46,49,53,56)$. Two studies $(48,53)$ based their intervention on social cognitive theory; two $(29,33)$ on empowerment model; one each based on supportive care model(46), information motivation and behavioral skill model(22), Hernandez theory of integration(23) and attribution theory(49). The mean duration of interventions was twelve weeks, ranging from two weeks $(49,55)$ to twelve months $(34)$. A wide range of intensity of intervention was reported ranging from 15-20 minutes over a day (31) to 1530 minutes over twelve months (34). Majority (68\%) of the studies administered both educational and behavioral components of intervention. The follow-up duration ranged from three months $(22,38,39,46,49$, $55,57,58)$ to eighteen months $(41)$. Five $(20 \%)$ studies used the multidisciplinary care approach to deliver the intervention $(29,32,44,46,50)$. The interventions were delivered in hospitals in $15(60 \%)$ studies whereas; community was the setting for nine (36\%) studies. One study reported administration of intervention both in hospital as well as community (43).

\subsection{Study Outcomes:}

\subsubsection{Primary Outcome (Glycemic Control HbA1c)}

$\mathrm{HbA} 1 \mathrm{c}$ was reported as an outcome measure in 23 studies. Majority $(70 \%)$ of the studies reported statistically significant reduction in $\mathrm{HbA} 1 \mathrm{c}$. Because of the significant heterogeneity ( $>50 \%$ ) among studies, random effect model was applied. At $95 \% \mathrm{Cl}$ with 3496 participants in 20 RCTs and 3 QESs, the magnitude of effect $-0.53(95 \% \mathrm{Cl}-0.73,-0.32)$ was statistically significant $(\mathrm{p}<0.00)$ showing a substantial reduction in $\mathrm{HbA} 1 \mathrm{c}$ in the experimental group compared to the control group. Pooled effect size of HbA1c is shown in Figure-2. The likelihood of publication bias among studies was measured with Funnel plot illustrated in Figure-3. The visual inspection of funnel plot shows an asymmetric appearance with a gap in the bottom indicating that there might be some publication bias; most likely for the reason that smaller studies without statistically significant effects remain unpublished.

\subsubsection{Stratified Analysis for change in HbA1c}

\section{a. Stratified analysis based on quality of the studies}

Based on the judgement of low, high and unclear risk of bias, a stratified analysis was performed by grouping studies into three categories: (1) low risk, (2) high risk, and (3) unclear risk. Studies with low risk of bias produced largest effect size $(-0.67 ; 95 \% \mathrm{Cl}-0.09,-0.24)$ compared to the studies with high risk (-0.57; $95 \% \mathrm{Cl}-0.93,-0.22)$ and unclear risk $(-0.25 ; 95 \% \mathrm{Cl}-0.40,-0.09)$. Overall heterogeneity was $87 \%$ therefore; a random effect model was applied (see Fig. $4 \mathrm{a})$.

\section{b. Stratified analysis based on components of intervention}

Earlier Gary TL et al., (2003) in their meta-analysis, concluded that studies with behavioral component of intervention were found more effective in reducing HbA1c compared to studies involved educational component only(24). In this analysis, studies were sub-grouped into two components of intervention; (1) educational and (2) combined educational and behavioral intervention. Pooled effect size indicated that studies with educational and behavioral components yielded larger effect size $(-0.59 ; 95 \% \mathrm{Cl}-0.86,-0.32)$ compared to studies with educational component only $(-0.42 ; 95 \% \mathrm{Cl}-0.56,-0.28)$. Overall heterogeneity $\left(\mathrm{I}^{2}\right)$ was $90 \%$ therefore; random effect model was applied (see Fig. 4b).

\section{b. Stratified analysis based on Duration of Intervention}

Existing evidence suggested that longer duration of intervention (> six months) showed significant reduction in HbA1c compared to shorter duration ( $<$ six months) (59). In this review considering the span of intervention; studies were sub-grouped into (1) studies with duration of intervention < three months; (2) three to six months; and (3) > six months. Pooled effect size indicated that studies with shorter duration (< three months) produced larger effect size (-0.56; $95 \% \mathrm{Cl}-0.86,-0.27)$ compared to studies with duration of intervention three to six months $(-0.45 ; 95 \% \mathrm{Cl}-0.75,-0.15)$ and studies with longer duration of intervention > six months $(-0.10 ; 95 \% \mathrm{Cl}-0.35,-0.16)$. Overall heterogeneity $\left(\mathrm{I}^{2}\right)$ was $83 \%$ therefore; random effect model was applied (see Fig. $\left.4 \mathrm{c}\right)$.

\section{c. Stratified analysis based on provider of intervention}

Evidence suggests that a multidisciplinary team approach is more effective in improving HbA1c(60). Given this, a stratified analysis was performed by dividing studies into three groups based on the provider of intervention: (1) a nurse; (2) other professional such as a physician, nutritionist, pharmacist or 
community health worker; and (3) multidisciplinary team members ( $\geq 2$ disciplines). Pooled effect size indicated that studies involved nurse as a provider of intervention produced larger effect size $(-0.80 ; 95 \% \mathrm{Cl}-1.44,-0.16)$ compared to studies with other professional as provider $(-0.50 ; 95 \% \mathrm{Cl}-0.75,-0.24)$ and studies involving $\geq 2$ disciplines $(-0.36 ; 95 \% \mathrm{Cl}-0.63,-0.10)$. Overall heterogeneity $\left(\mathrm{I}^{2}\right)$ was $87 \%$ therefore; random effect model was applied (see Fig. $\left.4 \mathrm{~d}\right)$.

\section{d. Stratified analysis based on setting of intervention}

To see the effect of setting, a stratified analysis was performed by grouping studies into three categories: (1) hospital, (2) community, and (3) combined setting including both hospital and community. Studies with intervention delivered in community setting produced largest effect size (-0.65; $95 \% \mathrm{Cl}-0.10$, $-0.29)$ compared to hospital $(-0.49 ; 95 \% \mathrm{Cl}-0.77,-0.22)$ and combined setting $(-0.14 ; 95 \% \mathrm{Cl}-0.42,-0.14)$. Overall heterogeneity was $87 \%$ therefore; a random effect model was applied (see Fig. 4e).

\subsubsection{Secondary Outcomes}

\section{a. Diet Control}

Eleven studies $(22,31-33,35,36,39,40,46,49,61)$ reported on dietary outcomes where the majority $(73 \%$; 8 out of 11$)$ of the studies showed statistically significant improvement in diet control in the interventions group.

\section{b. Physical Activity}

Ten studies $(22,31,32,35,36,39,40,46,49,61)$ reported on physical activity or exercise outcome where the majority $(70 \%$; 7 out of 10$)$ of the studies $(22,31$, $35,39,49,54,58)$ showed significant improvement in physical activity in the intervention group.

\section{c. Medication Adherence}

Three studies $(35,40,56)$ reported on this outcome that showed statistically non-significant improvement in medication adherence in the intervention group.

\section{d. Foot care}

Ten studies $(22,30-32,35,36,40,43,49,61)$ reported on foot care outcome. Majority $(60 \% ; 6$ out of 10$)$ of the studies $(30-32,35,36,49)$ showed statistically significant improvement in foot care in the intervention group.

\section{Discussion}

This review aimed at evaluating the effectiveness of patient-centered self-management care interventions on self-care outcomes of adults with type 2 DM compared with usual care. The most important indicator of optimum management of DM is glycemic control (HbA1c). Therefore, the primary outcome of this review was glycemic control (HbA1c) whereas, changes in diet control, physical activity, medication adherence and foot care were the secondary outcomes.

To estimate the overall effect of intervention, a meta-analysis was performed to calculate the magnitude of effect size for change in $\mathrm{HbA1c}$. Pooled effect size indicated a statistically significant difference in $\mathrm{HbA} 1 \mathrm{c}$ between experimental and control group $-0.53(95 \% \mathrm{Cl}-0.73,-0.32)$. The findings of this review are similar to the previous meta-analysis by Gray et al., (2003) that reported statistically significate reduction in $\mathrm{HbA} 1 \mathrm{c}(-0.43 ; 95 \% \mathrm{Cl}-0.71,-0.15)(24)$. This review confirmed that the patient-centered self-management interventions are accompanied with a significant decrease in $\mathrm{HbA1}$. Since, $\mathrm{HbA} 1 \mathrm{c}$ is one of the important predictors of DM associated complications and a key therapeutic goal towards its effective self-management; findings of this review have some important implications for contemporary practice. Evidence suggests that $21 \%$ risk is reduced for any DM associated complication and its related deaths with $1 \%$ decline in $\mathrm{HbA} 1 \mathrm{c}(62)$. Thus, reduction in $\mathrm{HbA} 1 \mathrm{c}$ has clinical significance.

Further, a stratified analysis for change in $\mathrm{HbA} 1 \mathrm{c}$ was performed with regards to the quality of the included studies which showed larger effects among studies with high quality judgement (low risk of bias) further supporting the evidence. Stratified analyses were also performed with regards to various key aspects of intervention in order to ascertain key elements that might contribute towards effective self-management of type 2 DM. In this review, interventions involving educational and behavioral components, spanned over shorter (< three months) duration, provided by nurses and delivered in community settings; were found more effective as indicated by larger effect sizes. Some findings of this review are contrary to the previous meta-analysis by Gary et al., (2003) where interventions that involved longer duration (>3 months) and provided by physicians; were found more effective. It appears that interventions with longer duration may carry an element of fatigue due to long contact times which may produce lesser effect. There is a need to explore further the factors associated with longer duration interventions demonstrating an area of research. Moreover, findings of this review showing larger effects with nurses as providers of intervention emphasize the importance that nurses are uniquely positioned to bring their expertise and knowledge towards effective self-management of type 2 diabetes. This, again demonstrate an area of research to further investigate the clinical effectiveness of nurse-led interventions. The findings are consistent with previous meta-analysis with regards to the setting of intervention confirming that interventions delivered in community settings are more effective(24).

With regards to secondary outcomes, this review indicated that patient centered self-management care interventions are effective in improving patients' diet control, physical activity, and foot care. However, the effect on medication adherence was not found effective which may be due to the reason that only a few studies reported this outcome.

\section{Limitations And Strengths}

The limitations of this review consist of: only English language studies were included; selective reporting of the outcomes might have affected the findings; frequent methodological biases which were found in included studies; insufficient description of intervention in the included studies; not reporting of 
medications or any drug prescription information since medication intake may act as a confounder between the interventions and outcomes.

However, this review contained numerous strengths which include: rigorous reviewing methods; thorough search to capture all relevant information, explicit and reproducible eligibility criteria, stratified analysis which allowed answering of clinically relevant and important questions.

\section{Conclusion}

This review concludes providing evidence supporting that patient centered care for DM self-management is effective in improving glycemic control and selfcare behaviors in adults with type 2 DM. Moreover, some gaps were found that are needed to be addressed: (1) Medication adherence was reported by a few studies, (2) a few studies provided a thorough description with regards to intervention including intensity, duration, length of follow-up and theoretical background, (3) Behavioral component was not described in adequate detail with regards to the methods which were applied.

\section{Abbreviations}

AADE: American Association of Diabetes Educators

CINAHL: Cumulative Index to Nursing and Allied Health

DM: Diabetes Mellitus

IDF: International Diabetes Federation

QES: Quasi Experimental Study

RCT: Randomized Controlled Trial

WHO: World Health Organization

\section{Declarations}

\section{Acknowledgements:}

Not applicable

\section{Authors' Contributions:}

KA: Study conception and design, Literature retrieval, drafting of the manuscript.

KD: Data analysis \& interpretation, critical revision of the manuscript.

RG: Study conception, critical revision and final approval of the manuscript.

EF: Conceptualizing the review process, data analysis and interpretation, critical revision and final approval of the manuscript.

\section{Availability of data and materials:}

Data and materials are available from the corresponding author upon request.

\section{Competing Interests:}

The authors declare that they have no competing interests.

\section{Consent for publication:}

Not applicable

\section{Ethics Approval and consent to participate:}

Not applicable

Funding:

None

\section{References}

1. Organization WH. Classification of diabetes mellitus. 2019;

2. Association AD. 2. Classification and diagnosis of diabetes: standards of medical care in diabetes-2018. Diabetes Care. 2018;41(Supplement 1):S13-27.

3. Glovaci D, Fan W, Wong ND. Epidemiology of diabetes mellitus and cardiovascular disease. Curr Cardiol Rep. 2019;21(4):21. 
4. Harding JL, Pavkov ME, Magliano DJ, Shaw JE, Gregg EW. Global trends in diabetes complications: a review of current evidence. Diabetologia. 2019;62(1):3-16.

5. Saeedi P, Petersohn I, Salpea P, Malanda B, Karuranga S, Unwin N, et al. Global and regional diabetes prevalence estimates for 2019 and projections for 2030 and 2045: Results from the International Diabetes Federation Diabetes Atlas. Diabetes Res Clin Pract. 2019;157:107843.

6. Organization WH. World Health Organization Global Report on Diabetes. Geneva World Heal Organ. 2016;

7. Worldometer CC. Worldometer,(2020). 2020.

8. Adnan M, Aasim M. Prevalence of type 2 diabetes mellitus in adult population of pakistan: a meta-analysis of prospective cross-sectional surveys. Ann Glob Heal. 2020;86(1).

9. Basit A, Fawwad A, Baqa K. Pakistan and diabetes-A country on the edge. Diabetes Res Clin Pract [Internet]. 2019;147(November):166-8. Available from: https://doi.org/10.1016/j.diabres.2018.11.001

10. Eckerblad J. Symptom burden among people with chronic disease. 2015.

11. Williams R, Karuranga S, Malanda B, Saeedi P, Basit A, Besançon S, et al. Global and regional estimates and projections of diabetes-related health expenditure: Results from the International Diabetes Federation Diabetes Atlas. Diabetes Res Clin Pract. 2020;108072.

12. Zhao C, Wong L, Zhu Q, Yang H. Prevalence and correlates of chronic diseases in an elderly population: A community-based survey in Haikou. PLoS One. 2019;13(6):1-11.

13. Powers MA, Bardsley JK, Cypress M, Funnell MM, Harms D, Hess-Fischl A, et al. Diabetes self-management education and support in adults with type 2 diabetes: a consensus report of the American Diabetes Association, the Association of Diabetes Care \& Education Specialists, the Academy of Nutrition and Dietetics, the American Academy of Family Physicians, the American Academy of PAs, the American Association of Nurse Practitioners, and the American Pharmacists Association. Sci Diabetes Self-Management Care. 2021;47(1):54-73.

14. Haas L, Maryniuk M, Beck J, Cox CE, Duker P, Edwards L, et al. National standards for diabetes self-management education and support. Diabetes Educ. 2012;38(5):619-29.

15. Kalra S, Megallaa MH, Jawad F. Perspectives on patient-centered care in diabetology. J Midlife Health. 2012;3(2):93.

16. Ford RT. Teaching comprehensive treatment planning within a patient-centered care model. J Dent Educ. 1988;52(2):114-7.

17. Davis K, Schoenbaum SC, Audet A-M. A 2020 vision of patient-centered primary care. J Gen Intern Med. 2005;20(10):953-7.

18. Beck J, Greenwood DA, Blanton L, Bollinger ST, Butcher MK, Condon JE, et al. 2017 National standards for diabetes self-management education and support. Diabetes Educ. 2018;44(1):35-50.

19. Organization WH. Diabetes action now: an initiative of the World Health Organization and the International Diabetes Federation. 2004;

20. Mehravar F, Mansournia MA, Holakouie-Naieni K, Nasli-Esfahani E, Mansournia N, Almasi-Hashiani A. Associations between diabetes self-management and microvascular complications in patients with type 2 diabetes. Epidemiol Health. 2016;38.

21. Lin K, Park C, Li M, Wang X, Li X, Li W, et al. Effects of depression, diabetes distress, diabetes self-efficacy, and diabetes self-management on glycemic control among Chinese population with type 2 diabetes mellitus. Diabetes Res Clin Pract. 2017;131:179-86.

22. Gavgani RM, Poursharifi H, Aliasgarzadeh A. Effectiveness of Information-Motivation and Behavioral skill (IMB) model in improving self-care behaviors \& Hba1c measure in adults with type2 diabetes in Iran-Tabriz. Procedia - Soc Behav Sci. 2010;5:1868-73.

23. Jutterström L, Hörnsten, Sandström H, Stenlund H, Isaksson U. Nurse-led patient-centered self-management support improves HbA1c in patients with type 2 diabetes-A randomized study. Patient Educ Couns [Internet]. 2016;99(11):1821-9. Available from: http://dx.doi.org/10.1016/j.pec.2016.06.016

24. Gary TL, Genkinger JM, Guallar E, Peyrot M, Brancati FL. Meta-analysis of randomized educational and behavioral interventions in type 2 diabetes. Diabetes Educ. 2003;29(3):488-501.

25. Moher D, Liberati A, Tetzlaff J, Altman DG, Group P. Preferred reporting items for systematic reviews and meta-analyses: the PRISMA statement. PLoS med. 2009;6(7):e1000097.

26. Sterne JAC, Savović J, Page MJ, Elbers RG, Blencowe NS, Boutron I, et al. RoB 2: a revised tool for assessing risk of bias in randomised trials. bmj. 2019;366.

27. Sterne JAC, Hernán MA, Reeves BC, Savović J, Berkman ND, Viswanathan M, et al. ROBINS-I: a tool for assessing risk of bias in non-randomised studies of interventions. bmj. 2016;355.

28. Azami G, Soh KL, Sazlina SG, Salmiah M, Aazami S, Mozafari M, et al. Effect of a nurse-led diabetes self-management education program on glycosylated hemoglobin among adults with type 2 diabetes. J Diabetes Res. 2018;2018.

29. Ebrahimi H, Sadeghi M, Amanpour F, Vahedi H. Evaluation of empowerment model on indicators of metabolic control in patients with type 2 diabetes, a randomized clinical trial study. Prim Care Diabetes. 2016;10(2):129-35.

30. Williams GC, Lynch M, Glasgow RE. Computer-Assisted Intervention Improves Patient-Centered Diabetes Care by Increasing Autonomy Support. Heal Psychol. 2007;26(6):728-34.

31. Sacco WP, Malone JI, Morrison AD, Friedman A, Wells K. Effect of a brief, regular telephone intervention by paraprofessionals for type 2 diabetes. J Behav Med. 2009 Aug;32(4):349-59.

32. Ing CT, Zhang G, Dillard A, Yoshimura SR, Hughes C, Palakiko D-M, et al. Social Support Groups in the Maintenance of Glycemic Control after CommunityBased Intervention. J Diabetes Res. 2016;2016:7913258.

33. Cheng L, Sit JWH, Choi K chow, Chair S ying, Li X, Wu Y, et al. Effectiveness of a patient-centred, empowerment-based intervention programme among patients with poorly controlled type 2 diabetes: A randomised controlled trial. Int J Nurs Stud [Internet]. 2018;79(December 2016):43-51. Available from:

Page $11 / 15$ 
http://dx.doi.org/10.1016/j.ijnurstu.2017.10.021

34. Pérez-Escamilla R, Damio G, Chhabra J, Fernandez ML, Segura-Pérez S, Vega-López S, et al. Impact of a community health workers-led structured program on blood glucose control among Latinos with type 2 diabetes: The DIALBEST Trial. Diabetes Care. 2015;38(2):197-205.

35. Zheng F, Liu S, Liu Y, Deng L. Effects of an outpatient diabetes self-management education on patients with type 2 diabetes in China: A randomized controlled trial. J Diabetes Res. 2019;2019.

36. Hailu FB, Moen A, Hjortdahl P. Diabetes self-management education (DSME) - Effect on knowledge, self-care behavior, and self-efficacy among type 2 diabetes patients in Ethiopia: A controlled clinical trial. Diabetes, Metab Syndr Obes Targets Ther. 2019;12:2489-99.

37. Carter EL, Nunlee-Bland G, Callender C. A patient-centric, provider-assisted diabetes telehealth self-management intervention for urban minorities. Perspect Health Inf Manag. 2011;8.

38. Yuan C, Lai CWK, Chan LWC, Chow M, Law HKW, Ying M. The effect of diabetes self-management education on body weight, glycemic control, and other metabolic markers in patients with type 2 diabetes mellitus. J Diabetes Res. 2014;2014.

39. Abraham AM, Sudhir PM, Philip M, Bantwal G. Efficacy of a Brief Self-management Intervention in Type 2 Diabetes Mellitus: A Randomized Controlled Trial from India. Indian J Psychol Med. 2020;XX(X):025371762093225.

40. Varming AR, Rasmussen LB, Husted GR, Olesen K, Grønnegaard C, Willaing I. Improving empowerment, motivation, and medical adherence in patients with poorly controlled type 2 diabetes: A randomized controlled trial of a patient-centered intervention. Patient Educ Couns. 2019;102(12):2238-45.

41. Spencer MS, Kieffer EC, Sinco B, Piatt G, Palmisano G, Hawkins J, et al. Outcomes at 18 months from a community health worker and peer leader diabetes self-management program for Latino adults. Diabetes Care. 2018;41(7):1414-22.

42. Al Omar M, Hasan S, Palaian S, Mahameed S. The impact of a self-management educational program coordinated through whatsapp on diabetes control. Pharm Pract (Granada). 2020;18(2):1-9.

43. Forjuoh SN, Bolin JN, Huber JCJ, Vuong AM, Adepoju OE, Helduser JW, et al. Behavioral and technological interventions targeting glycemic control in a racially/ethnically diverse population: a randomized controlled trial. BMC Public Health. 2014 Jan;14:71.

44. Kinmonth AL, Woodcock A, Griffin S, Spiegal N, Campbell MJ. Randomised controlled trial of patient centred care of diabetes in general practice: impact on current wellbeing and future disease risk. Bmj. 1998;317(7167):1202-8.

45. Scain SF, Friedman R, Gross JL. A structured educational program improves metabolic control in patients with type 2 diabetes. Diabetes Educ. 2009;35(4):603-11.

46. Taylor KI, Oberle KM, Crutcher RA, Norton PG. Promoting health in type 2 diabetes: Nurse-physician collaboration in primary care. Biol Res Nurs. 2005;6(3):207-15.

47. Windrum P, García-Goñi M, Coad H. The Impact of Patient-Centered versus Didactic Education Programs in Chronic Patients by Severity: The Case of Type 2 Diabetes Mellitus. Value Heal [Internet]. 2016;19(4):353-62. Available from: http://dx.doi.org/10.1016/j.jval.2016.01.014

48. Utz SW, Williams IC, Jones R, Hinton I, Alexander G, Yan G, et al. Culturally tailored intervention for rural African Americans with type 2 diabetes. Diabetes Educ. 2008;34(5):854-65.

49. Fardazar FE, Heidari $\mathrm{H}$, Solhi M. Effect of educational intervention based on locus of control structure of attribution theory on self-care behavior of patients with type II diabetes. Med J Islam Repub Iran. 2017;31(1):774-9.

50. Güner TA, Coşansu G. The effect of diabetes education and short message service reminders on metabolic control and disease management in patients with type 2 diabetes mellitus. Prim Care Diabetes. 2020;2-7.

51. Collaboration C. Review manager (RevMan)[computer program]. Copenhagen Nord Cochrane Cent. 2014;

52. Baker KA, Weeks SM. An overview of systematic review. J PeriAnesthesia Nurs. 2014;29(6):454-8.

53. Azami G, Soh KL, Sazlina SG, Salmiah MS, Aazami S, Mozafari M, et al. Effect of a Nurse-Led Diabetes Self-Management Education Program on Glycosylated Hemoglobin among Adults with Type 2 Diabetes. J Diabetes Res. 2018;2018.

54. Varming AR, Rasmussen LB, Husted GR, Olesen K, Grønnegaard C, Willaing I. Improving empowerment, motivation, and medical adherence in patients with poorly controlled type 2 diabetes: A randomized controlled trial of a patient-centered intervention. Patient Educ Couns. 2019 Dec;102(12):2238-45.

55. Zheng F, Liu S, Liu Y, Deng L. Effects of an outpatient diabetes self-management education on patients with type 2 diabetes in China: A randomized controlled trial. J Diabetes Res. 2019;2019:1073131.

56. Utz SW, Williams IC, Jones R, Hinton I, Alexander G, Yan G, et al. Culturally tailored intervention for rural African Americans with type 2 diabetes. Diabetes Educ. 2008;34(5):854-65.

57. Ebrahimi H, Seyedfatemi N, Namdar Areshtanab H, Ranjbar F, Thornicroft G, Whitehead B, et al. Barriers to Family Caregivers' Coping With Patients With Severe Mental Illness in Iran. Qual Health Res. 2018;

58. Hailu FB, Hjortdahl P, Moen A. Nurse-Led diabetes self-management education improves clinical parameters in Ethiopia. Front Public Heal. 2018;6(OCT):1-11.

59. Jeong S, Lee M, Ji E. Effect of pharmaceutical care interventions on glycemic control in patients with diabetes: A systematic review and meta-analysis. Ther Clin Risk Manag. 2018;14:1813-29.

60. Azami G, Soh KL, Sazlina S-G, Salmiah MS, Aazami S. Behavioral interventions to improve self-management in Iranian adults with type 2 diabetes: a systematic review and meta-analysis. J Diabetes Metab Disord. 2018;17(2):365-80.

61. Utz SW, Williams IC, Jones R, Hinton I, Alexander G, Yan G, et al. Culturally tailored intervention for rural African Americans with type 2 diabetes. Diabetes Educ. 2008;34(5):854-65.

Page 12/15 
62. Stratton IM, Adler Al, Neil HAW, Matthews DR, Manley SE, Cull CA, et al. Association of glycaemia with macrovascular and microvascular complications of type 2 diabetes (UKPDS 35): prospective observational study. Bmj. 2000;321(7258):405-12.

\section{Figures}

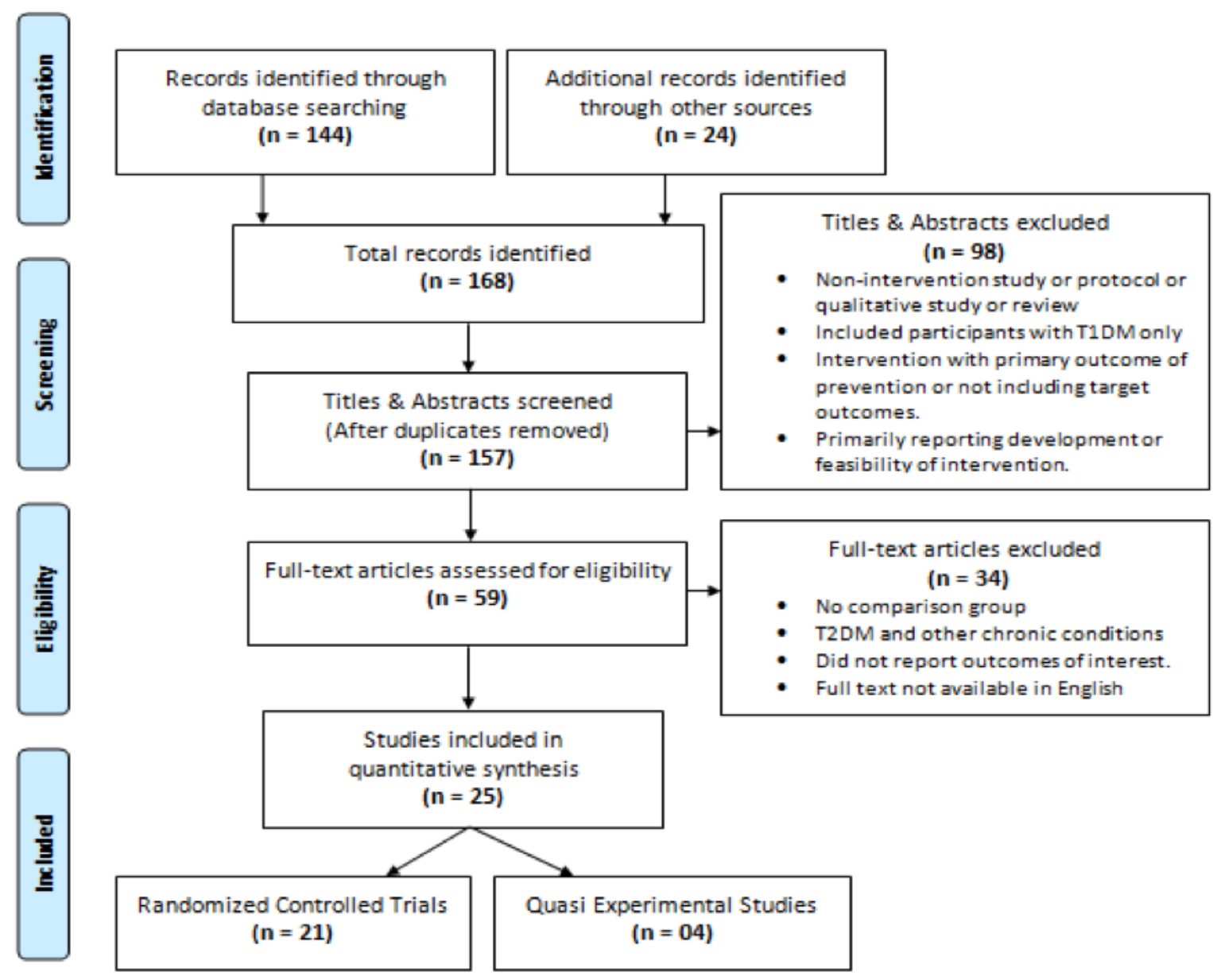

Figure 1

PRISMA flow chart for study selection and reasons for exclusion 


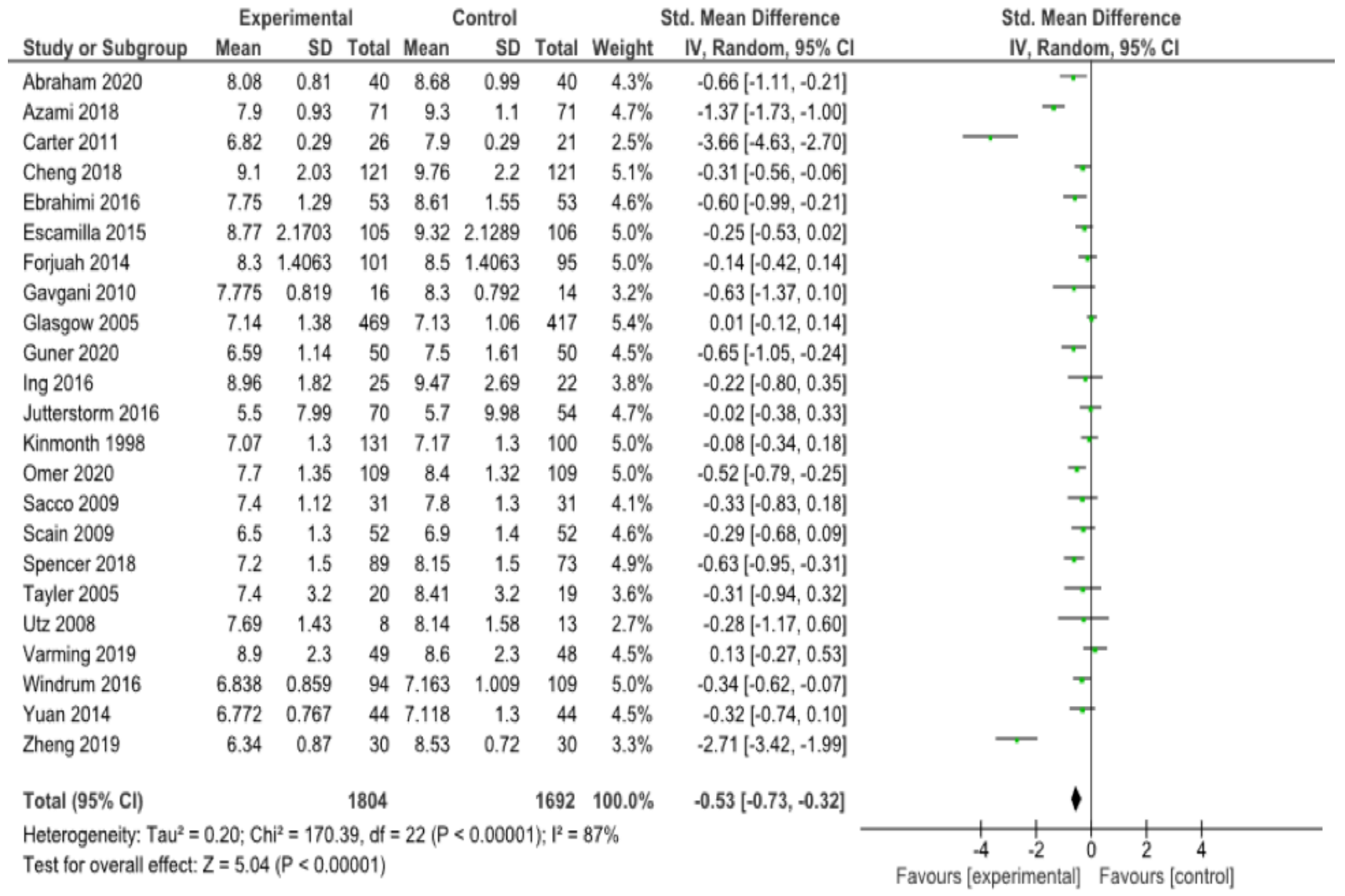

Figure 2

Forest Plot of pooled effect size of HbA1c

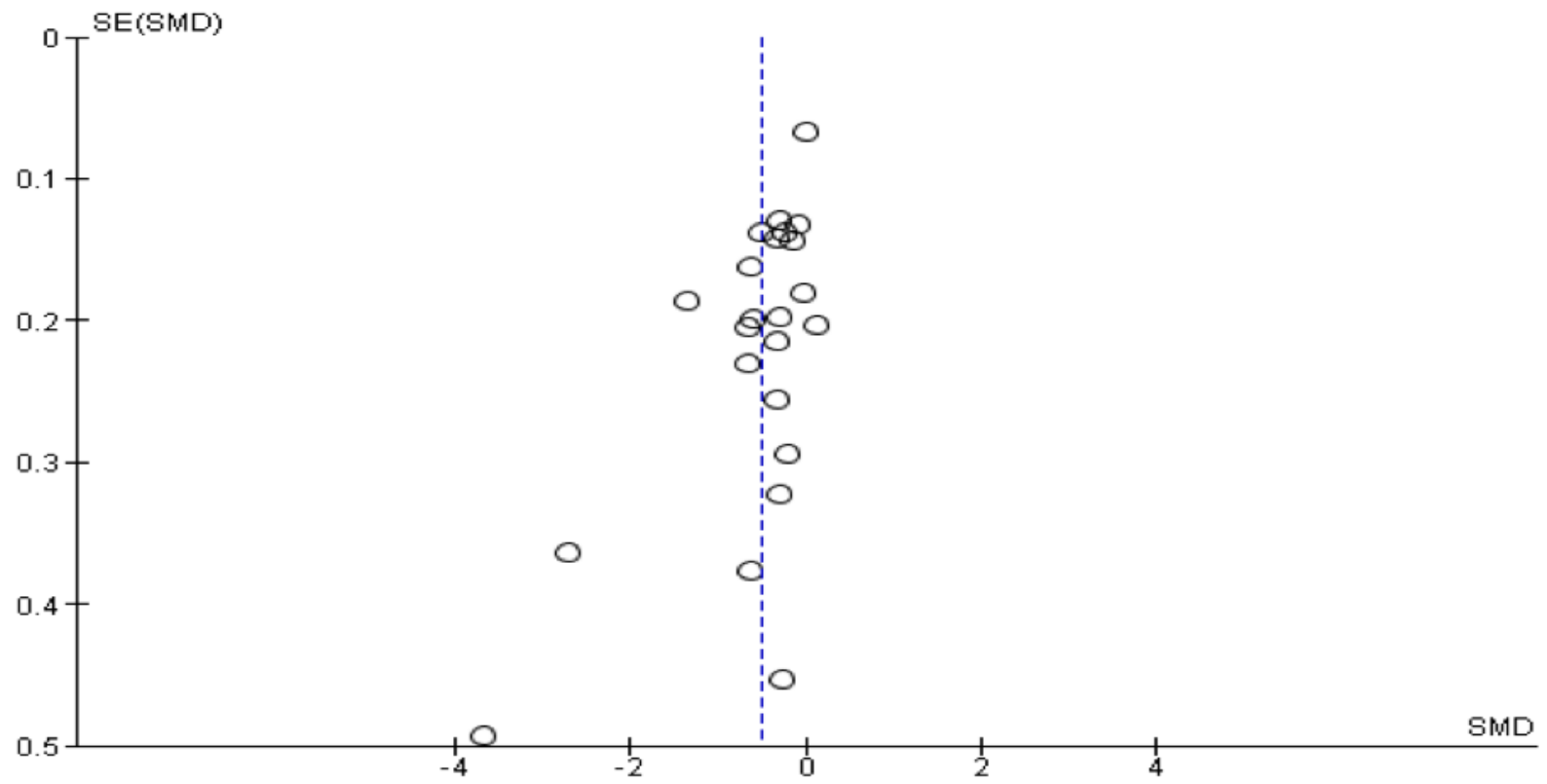

Figure 3

Funnel Plot for publication bias in HbA1c effects 


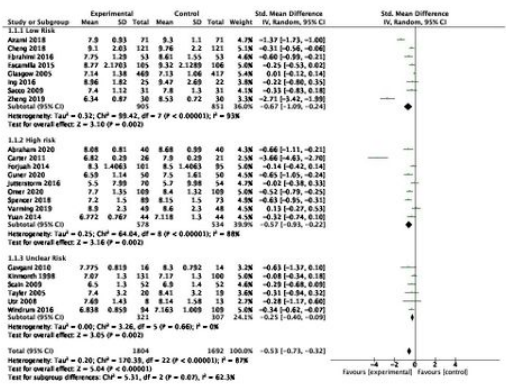

$4 \mathrm{a}$

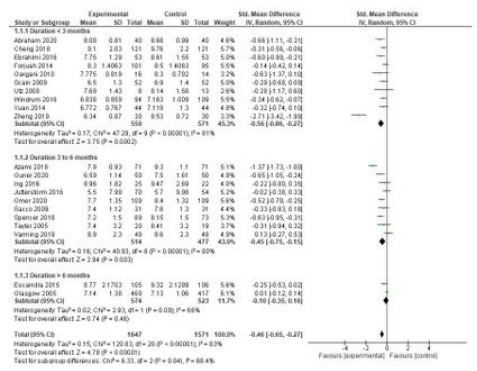

$4 c$

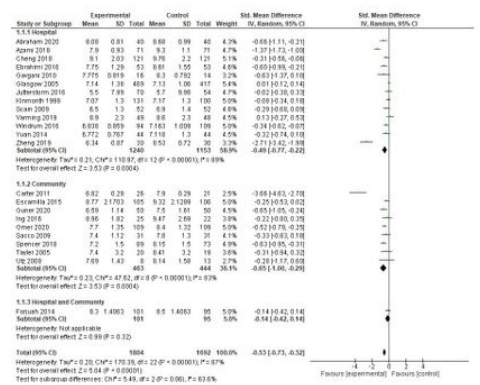

\section{Figure 4}

4a Pooled effect size of HbA1c in studies sub-grouped by quality. 4b Pooled effect size of HbA1c in studies sub-grouped by components of intervention. 4c Pooled effect size of HbA1c in studies sub-grouped by duration of intervention. 4d Pooled effect size of HbA1c in studies sub-grouped by Provider of intervention. 4e Pooled effect size of HbA1c in studies sub-grouped by setting of intervention.

\section{Supplementary Files}

This is a list of supplementary files associated with this preprint. Click to download.

- PrismaChecklist.KainatSR.doc

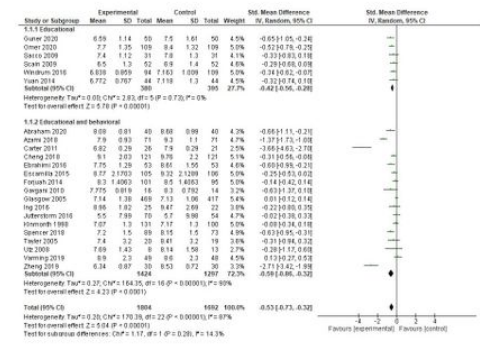

$4 b$

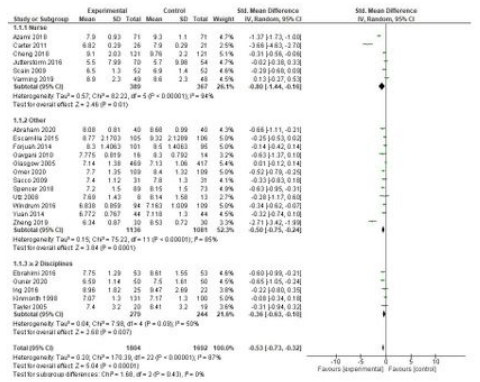

$4 d$ 